\title{
An Example of Principal Component Analysis Applied to Correlated Images
}

\author{
Anthony A. Maciejewski \\ Dept. of Electrical \& Computer Engineering \\ Purdue University, \\ 1285 Electrical Engineering Bldg., \\ West Lafayette, IN 47907-1285 USA
}

\author{
Rodney G. Roberts \\ Dept. of Electrical \& Computer Engineering \\ FAMU-FSU College of Engineering \\ 2525 Pottsdamer Street \\ Tallahassee, FL 32310-6046 USA
}

\begin{abstract}
The use of Principal Component Analysis (PCA), also known as Singular Value Decomposition (SVD), is a powerful tool that is frequently applied to the classification of hyperspectral images in remote sensing. Unfortunately, the utility of the resulting PCA may depend on the resolution of the original image, i.e., too coarse-grained of an image may result in inaccurate major principal components. This work presents an example of how the major principal component obtained from the PCA of a low-resolution image may be refined to obtain a more accurate estimate of the major principal component. The more accurate estimate is obtained by recursively performing a PCA on only those pixels that contribute strongly to the major principal component.
\end{abstract}

\section{INTRODUCTION}

Image analyses inherently require the handling of large volumes of information so that efficient mechanisms are required for the organization and classification of image data. One common reduced order representation for images, especially images that consist of multidimensional data, is based on the singular value decomposition (SVD). Variously referred to as eigendecomposition, principal component analysis (PCA) and Karhunen-Loeve transformation [1], the SVD has been successfully applied to a variety of imaging problems including face characterization [2] and recognition [3], lip-reading [4, 5], robot vision [6], video analysis [7], and remote sensing [8-10]. All of these applications are based on taking advantage of the fact that a set of highly correlated images can be approximately represented by a small set of singular vectors.

In this work, we consider the application of remote sensing; in particular, the analysis of hyperspectral images where there is multidimensional data (the spectral reflectance at various different wavelengths) associated with every pixel in an image. While PCA is commonly used in the representation and classification of such images, the singular vectors resulting from the analysis depend on the resolution of the original image. In general, the singular vectors obtained from higher-resolution images are more useful than those from lower-resolution images. We illustrate a technique by which the singular vector associated with the maximum singular value for a high-resolution hyperspectral image can be approximated from a lower-resolution image. An overview of PCA for hyperspectral images along with our proposed technique is presented in the following section. Section III presents an example of applying our approach to a specific hyperspectral image. Finally, the conclusions of this work are presented in Section IV.

\section{APPROACH}

Let $f_{i}$ represent an $m$-dimensional vector that corresponds to the spectral reflectance at pixel $i$. A hyperspectral image can then be represented by the matrix $F=\left[f_{l}, \ldots, f_{n}\right]$ where $n$ is the total number of pixels in the image. Let the SVD of $F$ be

$$
F=U \Sigma V^{T}
$$

where $U$ and $V$ are orthogonal, and $\Sigma$ is a matrix with nonzero terms appearing only on the main diagonal, and in descending order. The columns of $U$, denoted $u_{i}$, are referred to as the left singular vectors of $F$; these provide an orthonormal basis for the spectral reflectance, ordered in terms of importance. The corresponding singular values, which are nonnegative, measure how "aligned" the spectral signatures of the pixels are with the associated left singular vector. Thus, the first left singular vector can be interpreted as the spectral signature containing the "most common" information from all of the pixels in the image. Likewise, the first $k$ left singular vectors provide a basis for the best $k$-dimensional representation of the spectral response for the entire image. Thus the SVD provides a natural, ordered hierarchy for the compressed representation of information to within a user-defined level of accuracy. In addition to the left singular vectors, the information contained in the vectors comprising $V$ (referred to as the right singular vectors and denoted $v_{i}$ ) also provide useful information for analyzing hyperspectral images. Specifically, the components of $v_{i}$ measure how much each individual pixel contributes to the spectral signature represented by $u_{i}$.

Now consider a lower-resolution version of the hyperspectral image $F$, denoted by $F$. Let the images $F$ and $F^{\prime}$ 'represent the same total area, but with a pixel in $F^{\prime}$ 'being 
four times the area of a pixel in $F$. The lower resolution image can then be approximately represented by

$$
F^{\prime}=F G
$$

where $G$ is an $n$ by $n / 4$ matrix whose $i$ th column is a vector containing all zeros except for the four entries in the $4(i-1)+1$ to the $4(i-1)+4$ location which contain $1 / 4$. In other words, each pixel $f_{i}^{\prime}$ in the lower-resolution image is the average of four neighboring pixels in the higher-resolution image. Clearly, the SVD of $F^{\prime}$ will be different from that of $F$, which is arguably more useful because it represents more information. The question considered here is: Can one estimate the SVD of $F$ when only $F$ ' is available?

To estimate the most significant spectral signature in $F$, i.e., the vector $u_{l}$, we use the following approach: First we calculate the SVD of the complete low-resolution image $F$ '. We then examine the resulting maximum left singular $v_{l}$ and identify those elements, i.e., pixels, that have the largest value. We then construct a new matrix consisting of only these pixels and then compute its SVD. This process is then repeated until the resulting maximum left singular vector has converged or until a minimum number of pixels has been selected. Intuitively, one can expect this procedure to converge to a reasonable approximation of $u_{1}$ if those pixels that have a large component in $v_{l}^{\prime}$ come from areas in the image that are relatively homogeneous and have a strong contribution to $v_{l}$. The following section presents an example of such a case.

\section{RESULTS}

The hyperspectral image used in this study was produced by the MODIS/ASTER (MASTER) sensor during flight \#99005-06 on June 3, 1999 over Albuquerque and Sevilleta, NM [11]. The sensor produces a 50-band image covering the spectrum from 0.4-13 micrometers. The spectral characteristics of the sensor are given in Table 1. This investigation focused on classification of the groundcover in an area near Ballenger Ranch, south of Edgewood, NM. This area was manually classified using aerial photographs and ground surveys into two main groups, i.e., either dominated by woodland or dominated by grasses. These categories were further subcategorized as either heavy or light woodland (classes 1 and 2 respectively) or pinon-juniper savanna, shrub, or grassland (classes 3, 4, and 5 respectively). This classification is shown in Fig. 1 with statistics presented in Table 2.

A principal component analysis was performed on a 20 meter resolution version of the MASTER image that was registered to correspond to the classified image in Fig. 1. The first two most significant spectral signatures, i.e., the left singular vectors associated with the two largest singular values, are shown in Fig. 2. A scatter plot of the pixels from the MASTER image projected on to these first two principal components is given in Fig. 3. From this scatter plot, it is clear that the value of the projection onto the first principal component is a good indicator of the type of groundcover, i.e., a large value indicates grassland whereas a small value
TABLE 1

SPECTRAL CHARACTERISTICS OF THE MASTER SENSOR

\section{BAND HALFPP HALFPP FWHM CENTER PEAK}

\begin{tabular}{|c|c|c|c|c|c|}
\hline 01 & 0.4373 & 0.4812 & 0.0439 & 0.4592 & 0.4600 \\
\hline 02 & 0.4786 & 0.5213 & 0.0427 & 0.5000 & 0.5000 \\
\hline 03 & 0.5202 & 0.5628 & 0.0426 & 0.5415 & 0.5420 \\
\hline 04 & 0.5616 & 0.6026 & 0.0409 & 0.5821 & 0.5820 \\
\hline 05 & 0.6320 & 0.6913 & 0.0593 & 0.6616 & 0.654 \\
\hline 06 & 0.6916 & 0.7333 & 0.0416 & 0.7125 & 0.7120 \\
\hline 07 & 0.7307 & 0.7728 & 0.0421 & 0.7517 & 0.7520 \\
\hline 08 & 0.7810 & 0.8233 & 0.0423 & 0.8021 & 0.8020 \\
\hline 09 & 0.8486 & 0.8907 & 0.0421 & 0.8697 & 0.870 \\
\hline 10 & 0.8862 & 0.9271 & 0.0408 & 0.9066 & 0.908 \\
\hline 11 & 0.9260 & 0.9665 & 0.0404 & 0.9462 & 0.948 \\
\hline 12 & 1.5876 & 1.6418 & 0.0542 & 1.6147 & 1.6140 \\
\hline 13 & 1.6432 & 1.6981 & 0.0549 & 1.6707 & 1.6700 \\
\hline 14 & 1.6984 & 1.7515 & 0.0532 & 1.7249 & 1.7240 \\
\hline 15 & 1.7520 & 1.8075 & 0.0555 & 1.7797 & 1.7800 \\
\hline 16 & 1.8061 & 1.8593 & 0.0531 & 1.8327 & 1.8320 \\
\hline 17 & 1.8558 & 1.9101 & 0.0543 & 30 & 1.8780 \\
\hline 18 & 1.9059 & 1.9613 & 0.0554 & 1.9336 & 1.9340 \\
\hline 19 & 1.9555 & 2.0096 & 0.0541 & 1.9825 & 1.9820 \\
\hline 20 & 2.0583 & 2.1118 & 0.0 & 2.0851 & 2.0860 \\
\hline 21 & 2.1381 & 2.1916 & 0.0536 & 2.1649 & 2.1660 \\
\hline 22 & 2.1873 & 2.2422 & 0.0549 & 2.2147 & 2.2160 \\
\hline 23 & 2.2375 & 2.2903 & 0.0528 & 2.2639 & 2.2640 \\
\hline 24 & 2.2975 & 2.3677 & 0.0701 & 2.3326 & 2.3280 \\
\hline 25 & 2.3664 & 2.4317 & 0.0653 & 2.3990 & 2.3960 \\
\hline 26 & 3.0472 & 3.2041 & 0.1570 & 3.1257 & 3.1200 \\
\hline 2 & 3.2081 & 3.3548 & 0.1467 & 3.2815 & 3.2750 \\
\hline 28 & 3.3552 & 3.5119 & 0.1567 & 3.4336 & 3.4400 \\
\hline 29 & 3.5138 & 3.6683 & 0.1546 & 3.5911 & 3.5900 \\
\hline 30 & 3.6702 & 3.8093 & 0.1392 & 3.7398 & 3.7400 \\
\hline 31 & 3.8229 & 3.9769 & 0.1540 & 3.8999 & 3.9000 \\
\hline 32 & 3.9806 & 4.1324 & 0.1518 & 4.0565 & 4.0550 \\
\hline 33 & 4.1351 & 4.2880 & 0.1530 & 4.2116 & 4.2070 \\
\hline 34 & 4.2851 & 4.4380 & 0.1530 & 4.3616 & 4.3570 \\
\hline 35 & 4.4465 & 4.5911 & 0.1446 & 4.5188 & 4.5200 \\
\hline 36 & 4.5915 & 4.7478 & 0.1 & 4.6696 & 4.6700 \\
\hline 37 & 4.7494 & 4.9002 & 0.1508 & 4.8248 & 4.8250 \\
\hline 38 & 4.9042 & 5.0464 & 0.1422 & 4.9753 & 4.9750 \\
\hline 39 & 5.0481 & 5.2026 & 0.1544 & 5.1254 & 5.1250 \\
\hline 40 & 5.2046 & 5.3425 & 0.1379 & 5.2735 & 5.2750 \\
\hline 41 & 7.5724 & 7.9247 & 0.3523 & 7.7485 & 7.7926 \\
\hline 42 & 7.9271 & 8.3734 & 0.4463 & 8.1502 & 8.2048 \\
\hline 43 & 8.4203 & 8.7868 & 0.3665 & 8.6035 & 8.6247 \\
\hline 44 & 8.8268 & 9.2632 & & 9.0450 & 9.0543 \\
\hline 45 & 9.4762 & 9.8705 & 0.3942 & 9.6734 & 9.6654 \\
\hline 45 & 9.8933 & 10.2792 & 0.3859 & 10.0862 & 10.0845 \\
\hline 47 & 10.3130 & 10.9138 & 0.6009 & 10.6134 & 10.5542 \\
\hline 48 & 10.9570 & 11.6501 & 0.6931 & 11.3035 & 11.1838 \\
\hline 49 & 11.8480 & 12.3429 & 0.4949 & 12.0955 & 12.0740 \\
\hline 50 & 12.6152 & 13.1012 & 0.4861 & 12.8582 & 12.8455 \\
\hline
\end{tabular}

indicates woodland. Indeed, the spectral response of the principal left singular vector is dominated by those pixels that represent grassland. This can be clearly seen by comparing Fig. 4, which is a plot of the right singular vector associated with the maximum singular value, with the classified image in Fig. 1. 


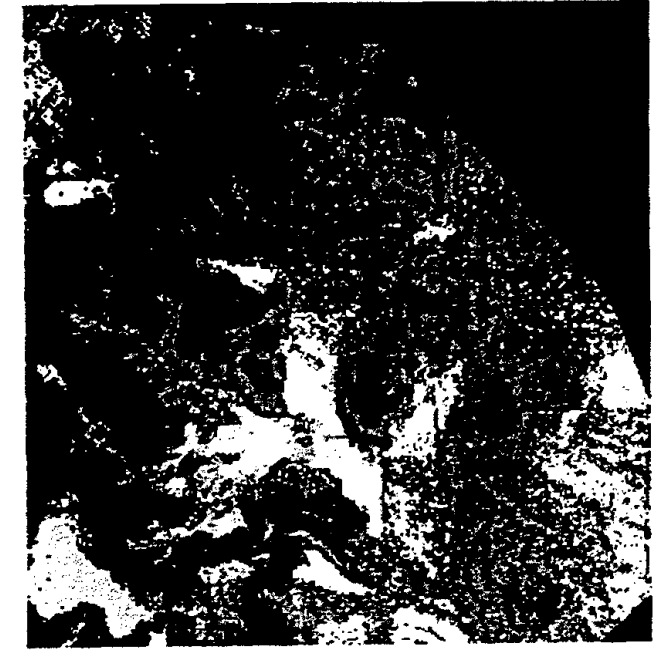

Figure 1 Classification of groundcover near Ballenger Ranch (south of Edgewood, NM). Darker shades of gray represent heavier groundcover.

TABLE 2

CLASSIFICATION HISTOGRAM

\begin{tabular}{lrr}
\multicolumn{1}{c}{ Class } & \multicolumn{2}{c}{ Area (number pixels) $\%$} \\
I Heavy Woodland & 20,152 & $31.6 \%$ \\
2 Light Woodland & 10,876 & $17.1 \%$ \\
Total Woodland (1 \& 2) & 31,028 & $48.7 \%$ \\
& & \\
3 Pinon-Juniper Savanna & 21,651 & $34.0 \%$ \\
4 Shrubs & 4,220 & $6.6 \%$ \\
5 Grasses & 6,866 & $10.8 \%$ \\
Total Grassland (3, 4, \& 5) & 32,737 & $51.3 \%$
\end{tabular}

Unfortunately, if this same procedure is performed on a MASTER image of lower resolution, the maximum spectral signature may not be as significantly dominated by the pixels primarily associated with grassland, and thus will not be as useful for classification. To illustrate this point, a principal component analysis was performed on an 80 -meter resolution MASTER image. The first three left singular vectors associated with the three largest singular values are plotted in Fig. 5. Note that the spectral characteristics of the first principal component differ significantly from those of the 20meter case (see Fig. 2). In particular, there are now peaks at bands 17,26 , and 34 that were not previously present and the sharp drop between bands 41 and 42 is no longer apparent. While there are still many predominantly grassland pixels that contribute to this first singular vector (see Fig. 6), there is also a significant contribution by pixels that are a mixture of woodland and grassland. Thus to recover the spectral signature of a predominantly grassland groundcover, one must use a linear combination of all three of the most significant left singular vectors. This is particularly

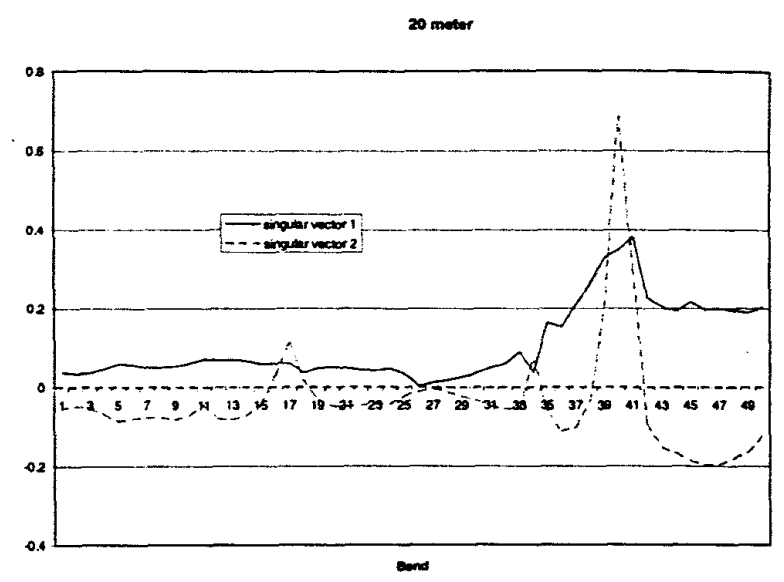

Figure 2 The two most significant spectral signatures, i.e., the singular vectors associated with the two largest singular values, for the 20 -meter resolution MASTER image.

\section{Scatter Plot of SV1 vs. SV2}

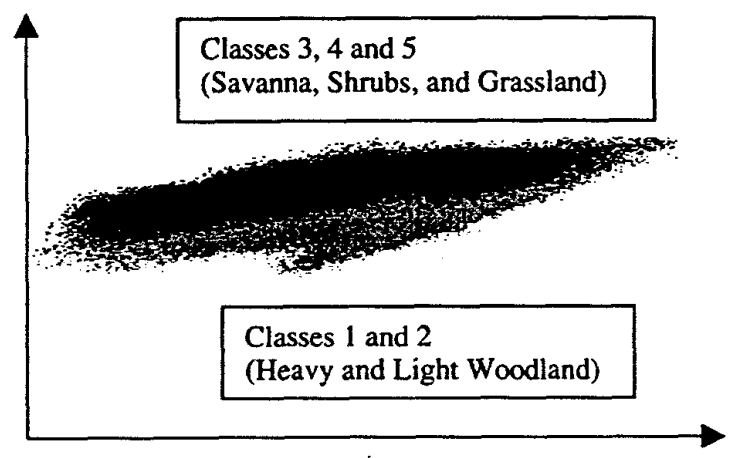

Figure 3 A scatter plot showing the projection of the 20-meter MASTER image onto the first two principal components.

noticeable by observing the strong negative peaks at bands 17,26 , and 34 of left singular vector three in Fig. 5 . The fact that left singular vector 3 plays a much larger role in determining the spectral signature of grassland in the low resolution PCA can be verified by comparing the right singular vectors for both the 20-meter case and the 80-meter case, shown in Figs. 7 and 8, respectively.

Clearly, the principal components of the low-resolution image are affected by having a much larger number of pixels that consist of mixed ground cover. To see if the maximum principal component obtained from the higher resolution PCA could be obtained by only using the lower resolution image, the procedure outlined in the previous section was performed. In particular, the pixels that had the largest contribution to the first principal component were extracted from the image. This corresponds to the top $10 \%$ of the brightest pixels in Fig. 6. A new PCA was performed on only these pixels. This recursive application of the PCA to selected regions of the 


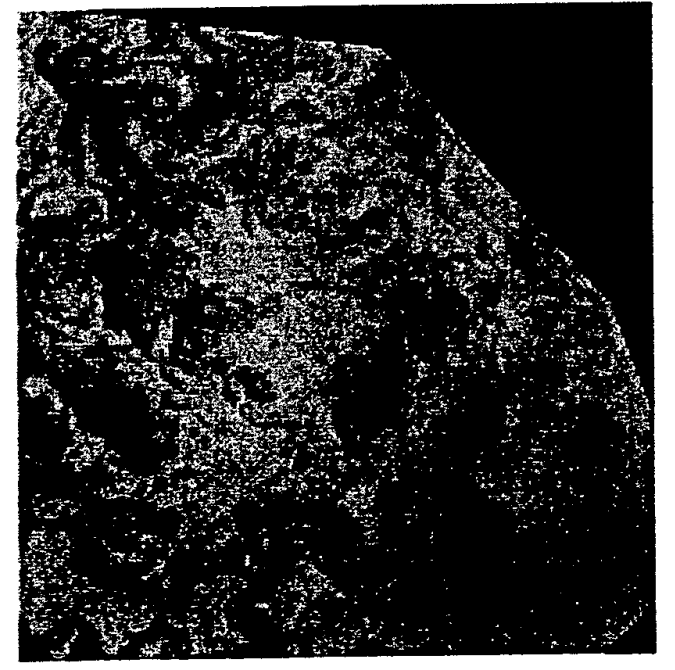

Figure 4 The right singular vector associated with the maximum singular value for the 20 -meter resolution MASTER image. The light regions indicate a strong contribution to the maximum left singular vector and by comparison to Fig. I clearly correspond to regions associated with grassland groundcover.

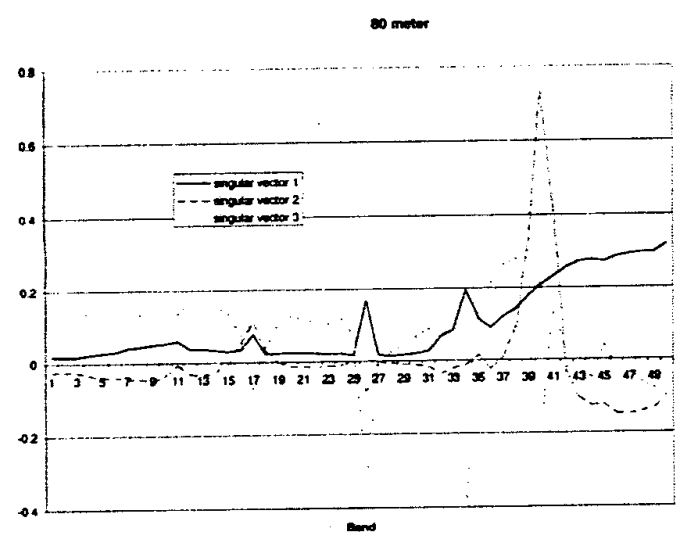

Figure 5 The three most significant spectral signatures, i.e., the singular vectors associated with the three largest singular values, for the 80-mete resolution MASTER image

image resulted in an increased contribution by pixels that were dominated by grassland. The resulting maximum left singular vector is shown in Fig. 9 and is very similar in spectral characteristics to the corresponding singular vector in the 20-meter case shown in Fig. 2. It is clearly superior to the maximum singular vector obtained from the initial PCA performed on the entire 80-meter image (shown in Fig. 5).

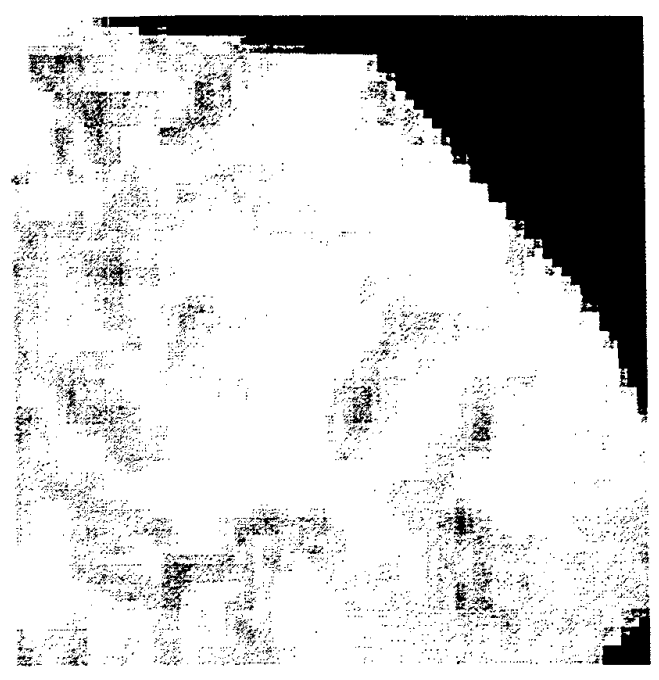

Figure 6 The right singular vector associated with the maximum singular value for the 80-meter resolution MASTER image. There is clearly a much larger contribution from areas that are not dominated by grassland as compared to the 20-meter case in Fig. 4.

\section{CONCLUSIONS}

This work has presented an example of a case where the left singular vector associated with the maximum singular value, obtained by performing a principal component analysis on a low resolution hyperspectral image, can be refined to approach the value obtained if one had a much higher resolution image. This is useful because the singular vector associated with a higher resolution image more accurately reflects the spectral characteristics of the most dominant feature in the image and is thus more useful for classification. The more accurate estimate of the maximum singular vector is obtained by recursively performing a PCA on selected portions of the low-resolution image. It has not been shown that this procedure always results in a more accurate estimate. Also, it may be possible to extend this procedure to obtain estimates of all significant singular vectors, in addition to the one associated with the maximum singular value. Both of these points are the subjects of our current work.

\section{ACKNOWLEDGMENTS}

This work was supported by the National Imagery and Mapping Agency under contract no. NMA201-00-1-1003. The authors gratefully acknowledge the assistance of Paul Neville and Sandra White of the Earth Data Analysis Center, The University of New Mexico, for providing the authors with the images and data for this work. The authors would also like to thank Dave Landgrebe and Larry Biehl of Purdue: University for providing the MultiSpec data analysis software: system that was used in much of the analysis. (The: MultiSpec software and documentation is available at http://dynamo.ecn.purdue.edu/ biehl/MultiSpec) 


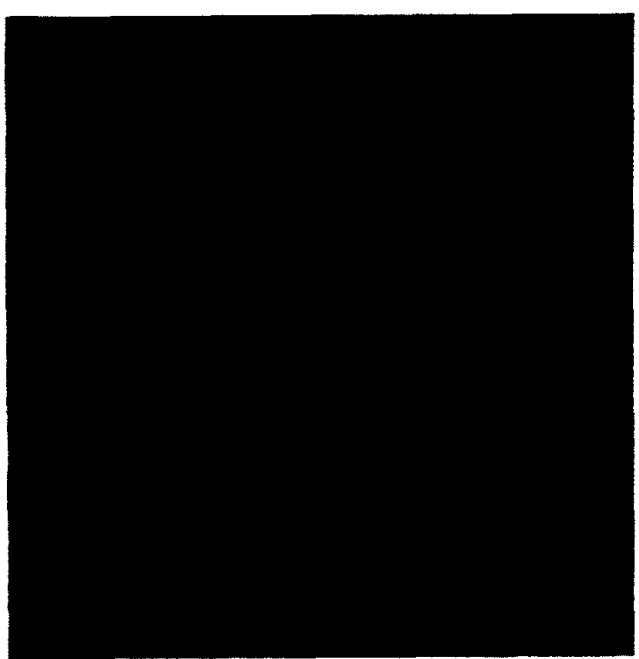

Figure 7 The right singular vector associated with the third largest singular value for the 20 -meter resolution MASTER image. Note that there is very little contribution of the associated left singular vector.

\section{REFERENCES}

[1] K. Fukunaga, Introduction to statistical pattern recognition, Academic Press, London, second edition, 1990.

[2] L. Siroyich and M. Kirby, "Low-dimensional procedure for the characterization of human faces," Journal of Optical Sociery of America, vol. 4, no. 3. pp. $519-524$, March 1987.

[3] M. Turk and A. Pentland. "Eigenfaces for recognition," Joumal of Cognitive Neuroscience, vol. 3, no. 1, pp. 71 -86, March 1991.

[4] H. Murase and R. Sakai. "Moving object recognition in eigenspace representation: Gait analysis and lip reading," Pattem Recognition Letters, vol. 17, no. 2. pp. 155-162. Feb. 1996.

[5] G. Chiou and J.-N. Hwang, "Lipreading from color video," IEEE Transactions on Image Processing, vol. 6, no. 8, pp. $1192-1195$, Aug. 1997.

[6] S. K. Nayar, S. A. Nene, and H. Murase, "Subspace method for robot vision," IEEE Transactions on Robotics and Automation, vol. 12, no. 5 , Oct. 1996.

[7] K. J. Han and A. H. Teuffik, "Eigen-image based video segmentation and indexing." Proc. IEEE International Conference on Image Processing., pp. 538 -54I. (Alamitos, CA, USA), 1997.

[8] X. Jia and J. A. Richards, "Segmented principal components transformation for efficient hyperspectral remote-sensing image display and classification," IEEE Trans. Geoscience and remote sensing. vol. 37, no. 1, Jan. 1999, pp. 538-54?.

[9] C-I. Chang, Q. Diu, T-L. Sun, and M.L.G. Althouse, "A joint band prioritization and band-decorrelation approach to band selection for hyperspectral image classification." IEEE Truns. Geoscience and remote sensing, vol. 37, no. 6. Nov. 1999. pp. 2631-2641.

[10| J. A. Richards and X. Jia. Remote Sensing Digital Image Analysis. Springer. NY 1999.

[11] hup:/nasterweh jol.nasa.gov/

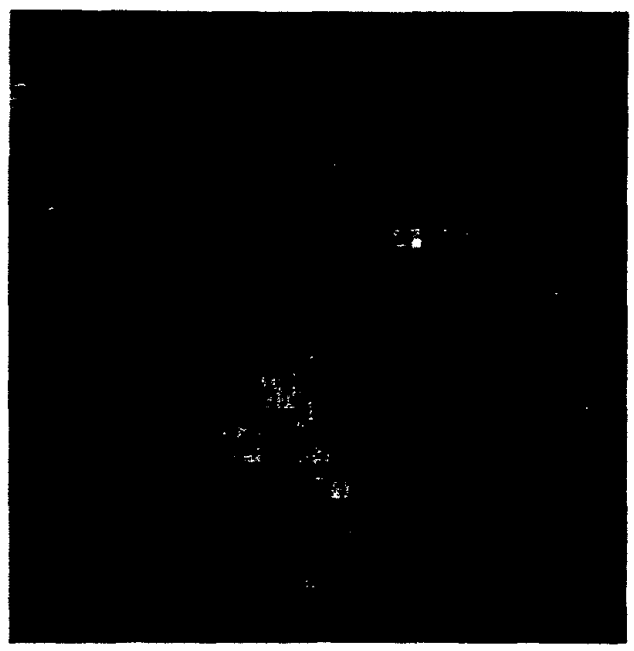

Figure 8 The right singular vector associated with the third largest singular value for the 80 -meter resolution MASTER image. Note that there is significantly more contribution of the associated left singular vector than with that of the 20-meter image shown in Fig. 7.

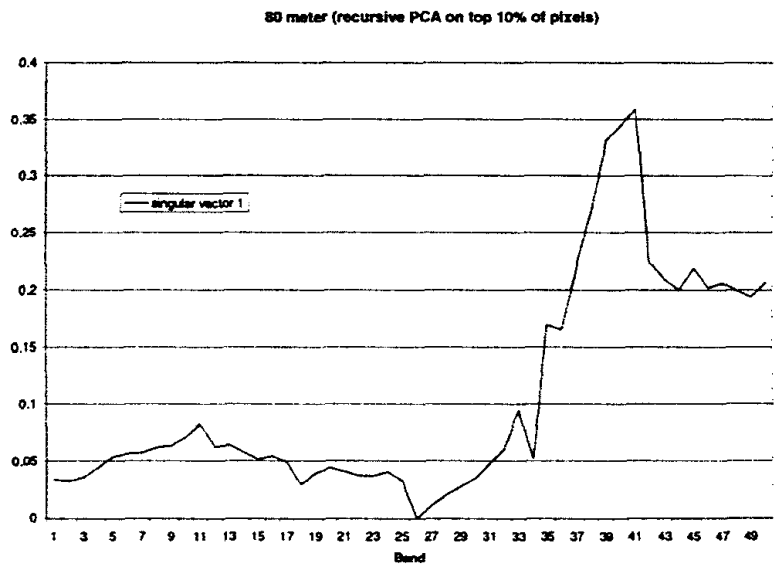

Figure 9 The left singular vector associated with the largest singular value for the $\mathbf{8 0}$ meter resolution MASTER image after recursively performing a PCA on the $10 \%$ of the pixels that had the highest conuribution to this principal component. Note that the spectral characteristics of this maximum left singular vector are very similar to that of the higher resolution image given in Fig. 2 . 